\title{
Imaging Spectroscopy on Preflare Coronal Nonthermal Sources Associated with the 2002 July 23 Flare
}

\author{
Ayumi Asai ${ }^{1}$, Hiroshi Nakajima ${ }^{1}$, Masumi Shimojo ${ }^{1}$, Takaaki Yokoyama ${ }^{2}$, Satoshi \\ Masuda $^{3}$, and Säm Krucker ${ }^{4}$ \\ asai@nro.nao.ac.jp
}

\begin{abstract}
We present a detailed examination on the coronal nonthermal emissions during the preflare phase of the X4.8 flare that occurred on 2002 July 23. The microwave (17 GHz and $34 \mathrm{GHz}$ ) data obtained with Nobeyama Radioheliograph, at Nobeyama Solar Radio Observatory and the hard X-ray (HXR) data taken with Reuven Ramaty High Energy Solar Spectroscopic Imager obviously showed nonthermal sources that are located above the flare loops during the preflare phase. We performed imaging spectroscopic analyses on the nonthermal emission sources both in microwaves and in HXRs, and confirmed that electrons are accelerated from several tens of $\mathrm{keV}$ to more than $1 \mathrm{MeV}$ even in this phase. If we assume the thin-target model for the HXR emission source, the derived electron spectral indices $(\sim 4.7)$ is the same value as that from microwaves $(\sim 4.7)$ within the observational uncertainties, which implies that the distribution of the accelerated electrons follows a single power-law. The number density of the microwave-emitting electrons is, however, larger than that of the HXR-emitting electrons, unless we assume low ambient plasma density of about $1.0 \times 10^{9} \mathrm{~cm}^{-3}$ for the HXR-emitting region. If we adopt the thick-target model for the HXR emission source, on the other hand, the electron spectral index $(\sim 6.7)$ is much different, while the gap of the number density of the accelerated electrons is somewhat reduced.
\end{abstract}

\footnotetext{
1 Nobeyama Solar Radio Observatory, National Astronomical Observatory of Japan, Minamimaki, Minamisaku, Nagano, 384-1305, JAPAN

2 Department of Earth and Planetary Science, University of Tokyo, Hongo, Bunkyo, Tokyo, 113-0033, JAPAN

3 Solar-Terrestrial Environment Laboratory, Nagoya University, Chikusa, Nagoya, Aichi, 464-8601, JAPAN

${ }^{4}$ Space Sciences Laboratory, University of California, Berkeley, CA94720, USA
} 
Subject headings: acceleration of particles — Sun: corona — Sun: flares — Sun: radio radiation — Sun: X-rays, gamma rays

\section{Introduction}

Nonthermal emissions from accelerated particles are often observed in hard X-rays (HXRs), $\gamma$-rays, and microwaves at the beginning of a solar flare. Although these nonthermal emissions are undoubtedly associated with intense energy release processes, the mechanisms to accelerate particles are still unclear, and they have been one of the most important and the most difficult problems in solar physics (see reviews by, e.g., Aschwanden 2002). The HXR nonthermal emission is well explained with the bremsstrahlung emission, which is emitted by the nonthermal electrons with energies $E \gtrsim 20 \mathrm{keV}$. In the microwave range, on the other hand, the gyrosynchrotron emission is the most promising nonthermal emission. The microwave-emitting electrons have relatively higher energies, such as sub-relativistic to relativistic energy. Although the emission mechanisms and electron energies are totally different, HXR and microwave emissions have shown a lot of similarities, especially in the lightcurves (e.g., Kundu 1961). The similarities have been thought to be evidences that microwave-emitting electrons are accelerated with the same mechanism as that for HXRemitting electrons. On the other hand, it has been also reported that an electron energy spectral index derived from HXR emissions is often larger (softer) than that derived from microwave emissions (e.g., Silva et al. 1997), and the temporal behaviors of the spectral indices are totally different between HXR and microwave. These suggest a possibility that spectra of nonthermal electrons have a bend, and that the high energy electrons that emit microwaves are accelerated more efficiently than the HXR-emitting electrons.

To explain the gap of spectral indices, several models have been suggested. For example, Somov \& Kosugi (1997) suggested that collapsing magnetic trap works efficiently for particle acceleration of higher energy electrons. Alternatively, Silva et al. (1997) suggested that we should take into account the transport mechanism of accelerated electrons, such as magnetic trapping, since the nature of the emission mechanisms is different for each emission range. In HXRs, nonthermal emissions from footpoints of flare loops are dominant, and these are described with "thick-target" bremsstrahlung emission model. However, the thick-target model only gives us a spectrum of the injected electron flux from a HXR emission spectrum at rather lower energy. Therefore, the conversion from the injected electron flux to the number of the nonthermal electrons is needed to compare it with the number of the microwaveemitting electrons at higher energy, and it requires another estimation such as the deflection 
time of precipitating electrons. If the deflection time has some dependences on electron energy, the resulting electron spectral indices could be modulated. Moreover, since the magnetic trapping works for a quite long time, and sometimes lasts several tens of minutes, the microwave spectra suffer modulations more, and it becomes more difficult to derive the information of the electron acceleration in the later phase.

Recently, we examined the HXRs features of the 2002 July 23 flare, and reported that the nonthermal energy even before the impulsive phase was quite large (Asai et al. 2006, Paper I). We refer to the time range when we can see the emission sources as "preflare phase". We found sufficient emissions both in HXRs and in microwaves that can be candidates for nonthermal emissions during the preflare phase. The emission sources both in HXRs and in microwaves were located on the flare loops, and the position almost corresponds to each other. Examining the imaging spectroscopic features of the emission sources both in HXRs and in microwaves and comparing the features with those of the peak time are required to know particle acceleration mechanism in the preflare phase. In order to derive information on the nonthermal electrons in the preflare phase, we examined in detail the features of the emission sources spatially, temporally, and spectroscopically. In this paper we report the results of the imaging spectroscopy on the coronal emission sources observed in HXRs and in microwaves during the preflare phase. In $\S 2$ we describe the observational data, and we discuss the density estimation in $\S 3$. In $\S 4$ we present the detailed reports on the imaging spectroscopy of the coronal emission sources both in microwaves and in HXRs. In $\S 5$ we summarize our results and offer discussions.

\section{Observations}

The intense solar flare (X4.8 on the GOES scale) occurred in NOAA Active Region 10039 (S12 ${ }^{\circ}, \mathrm{E}^{\circ}{ }^{\circ}$ ) on 2002 July 23. The Reuven Ramaty High Energy Solar Spectroscopic Imager (RHESSI: Lin et al. 2002) showed us many spectacular features in HXR and $\gamma$ ray wavelengths (e.g., Lin et al. 2003a). This flare was also observed in microwaves with the Nobeyama Radioheliograph (NoRH: Nakajima et al. 1994), as reported by White et al. (2003). The start time of this flare was recorded as 00:18 UT from the GOES flux, and the impulsive phase that is defined by the intense nonthermal emissions in HXRs and in microwaves started at about 00:27:30 UT. Figure 1 shows time profiles of the flare in SXRs, microwaves, and HXRs. The top panel shows the lightcurves in SXRs taken with the GOES $1.0-8.0 \AA$ (top) and $0.5-4.0 \AA$ (bottom) channels. The bottom three panels of Figure 1 show the expanded lightcurves of the rise phase of the flare (from 00:12 UT to 00:30 UT), which corresponds to the time between the two dashed lines in the top panel. The top of 
the three panels shows the lightcurves of the GOES $1.0-8.0 \AA$ and $0.5-4.0 \AA$ channels, the middle panel shows the ones at NoRH $17 \mathrm{GHz}$ and $34 \mathrm{GHz}$, and the bottom one shows the RHESSI time profiles in three energy ranges of $12-25,25-40$, and $60-100 \mathrm{keV}$. In this paper we focus on the nonthermal emissions in HXRs and in microwaves of the preflare phase, from 00:22:30 to 00:24:06 UT, on 2002 July 23, which corresponds to the time between the two dotted lines in the bottom three panels. This time range corresponds to the Phase II and III in Paper I, and we can identify the first nonthermal emissions and the onset of the faint ejection in the extreme ultraviolet images during this phase.

Figure 2 presents a microwave spectrum taken at 00:23:59 UT by the Nobeyama Radio Polarimeters (NoRP; Torii et al. 1979; Shibasaki et al. 1979; Nakajima et al. 1985). NoRP measure the total fluxes of the flare at 1, 2, 3.75, 9.4, 17, 35 and $80 \mathrm{GHz}$ with a temporal resolution of 0.1 second. We fitted the spectrum by using the NoRP data of $2,3.75,9.4,17$ and $35 \mathrm{GHz}$, and obtained the spectral index $\alpha\left(F_{\nu} \propto \nu^{\alpha} ; F_{\nu}\right.$ is the flux density at frequency $\nu$ ) of -2.65 and the turn-over frequency of about $9 \mathrm{GHz}$. As is seen in Figure 1 and will be mentioned more below, during the time range on which we focus, the thermal emission rapidly increases and the thermal component also emits microwaves. We estimated the microwave thermal emission observed with NoRP at 17 and $35 \mathrm{GHz}$ by using the GOES data and it is about 2 SFU. Since this somewhat hardens the microwave spectrum, we modified the NoRP spectrum as shown with the dashed line in Figure 2, and the fitted spectral index $\alpha$ in the optically-thin part is a little steepened to be about -2.95 . We re-plotted the fitting result on Figure 2 with the gray dashed line.

On the other hand, NoRH observes the Sun at two frequencies, 17 and $34 \mathrm{GHz}$ during Japanese daytime (normally from 22:45 to 06:30 UT) with a temporal resolution of 1 second. We can derive a spectral index $\alpha$ by using the flux ratio. The derived index $\alpha$ is for the optically-thin gyrosynchrotron emission, since both the 17 and $34 \mathrm{GHz}$ emissions are in the optically-thin part. The spatial resolutions (FWHMs of the synthesized beam) of NoRH data are $14^{\prime \prime}$ for $17 \mathrm{GHz}$ and $7^{\prime \prime}$ for $34 \mathrm{GHz}$.

Lin et al. (2003b) and Holman et al. (2003) already reported on the HXR emission in the preflare phase, although their works were based on the spatially integrated emission. We also synthesized the HXR images obtained with RHESSI by using grids $3-8$ and Clean method, which gives the spatial resolution (FWHM) of about 10". EUV images of the flare were obtained with the Transition Region and Coronal Explorer (TRACE; Handy et al. 1999; Schrijver et al. 1999). We used $195 \AA$ images, in which the Fe XII line formed at $\sim 1 \mathrm{MK}$ is normally dominant. The pixel size of the CCD is $1^{\prime \prime} 0$, and the temporal resolution is about 9 second.

Here, we summarize the spatial features of the emission sources. Figure 3 shows images 
of the phase in EUV, HXR, and microwave. The panel (a) is the TRACE $195 \AA$ image taken at 00:24:31 UT. We can see a large two-ribbon structure that brightens from 00:20 UT. We can also see a diffuse loop-like structure that is identified as Fe XXIV emission from 20 MK plasma, as is often observed in TRACE $195 \AA$ images during the impulsive phase of a flare. The diffuse loop seems to connect the two-ribbon structure as shown in the cartoon of the panel (d). We overlaid a microwave contour images of NoRH $34 \mathrm{GHz}$ on each panel with the white dashed line (while it is the gray dashed line in the panel $\mathrm{d}$ ). We also overlaid the NoRH $17 \mathrm{GHz}$ contour image on the panel (b) with the gray solid line. The levels of the contours are 20,40,60, $80 \%$ of the maximum intensity, and only the highest level $(80 \%)$ of the $34 \mathrm{GHz}$ contours is shown with the solid line in each panel. In addition, on panels (a), (b), and (d), we overlaid an HXR contour image observed with RHESSI in $30-45 \mathrm{keV}$ with the black solid line. The levels of the contours are 40,60, 80, $95 \%$ of the maximum intensity. We recognize a microwave and an HXR emission sources appear above the flare ribbons (Lin et al. 2003b). The position of the microwave emission source is slightly lower than that of the HXR emission source $\left(\sim 10^{\prime \prime}\right)$, which corresponds to the top of the post flare loops that become visible in the later phase in the TRACE images (see also Paper I). The footpoint emission sources are much weaker and unclearer than the loop-top sources. We can see compact emission sources on the western flare ribbon both in HXRs and in microwaves, and we can marginally see the extension of the HXR contour line that outlines the eastern flare ribbon. The microwave contour image with the lowest level also outlines a large loop-like bright region in the $\mathrm{NoRH}$ image. This remains visible for several hours before this flare started (White et al. 2003).

\section{Density Estimation}

Here, we present the density estimation of the ambient corona where the nonthermal emission sources appear. Before the flare in question started (from 22:00 UT on 2002 July $22)$, the GOES SXR fluxes increased, and this probably comes from the emission source which is visible as the large loop-like bright region in the microwave (White et al. 2003). This feature seen in the before-the-flare phase could be related to a small flare that occurred at 22:00 UT in the same active region, although we could not confirm this due to a lack of image data for the small event. We estimated the temperature and the emission measure of this emission source, by using the ratios of the two of GOES channels, and by defining the emission from the background corona as the average emission between 20:30 and 21:10 UT on 2002 July 22. Then, the temperature and the emission measure are estimated to be about 5.7 MK and $8.0 \times 10^{48} \mathrm{~cm}^{-3}$ at 23:30 UT, respectively. As we already reported in Paper I, we could estimate the microwave fluxes in 17 and $34 \mathrm{GHz}$ from the temperature and the 
emission measure, by assuming that the free-free emission is dominant for the microwave source, and confirmed that they were almost the same as the observed values.

After 00:18 UT, on the other hand, the GOES temperature rapidly increases and is higher than $10 \mathrm{MK}$ after 00:21 UT. This means that hot thermal plasma ( $T>10 \mathrm{MK})$ is also generated as well as the nonthermal emissions seen in HXRs and in microwaves. The accelerated nonthermal electrons probably traveled in this hot plasma, and therefore, the density estimation of the hot plasma is required for further discussions. To derive the temperature and the emission measure of the hot component, we re-defined the emission from the background corona as the emission between 23:30 and 00:10 UT. They are estimated about $15 \mathrm{MK}$ and $2.4 \times 10^{48} \mathrm{~cm}^{-3}$ at 00:23 UT. To derive the ambient plasma density, we assume the source volume. The size of the HXR source is about $27^{\prime \prime}$ (in east-west direction) $\times$ $20^{\prime \prime}$ (in north-south direction). Hence, assuming the line-of-sight depth of the emission source as $1.5 \times 10^{9} \mathrm{~cm}$, the volume and the density are about $4.2 \times 10^{27} \mathrm{~cm}^{3}$ and $2.4 \times 10^{10} \mathrm{~cm}^{-3}$.

\section{Imaging Spectroscopy}

\subsection{Microwave Emission Source}

First, we examine the spectral features of the microwave emission source by using the NoRH data. For the analyses, we synthesized the images both at 17 and $34 \mathrm{GHz}$ from 00:23:00 to 00:23:40 UT (40 second duration) every 1 second, and integrated them to make one image at each frequency. Then, we conformed the beam size of the $34 \mathrm{GHz}$ image to that of the $17 \mathrm{GHz}$ image to take ratio of the intensities, by convolving each image by the beam of the other image. Roughly speaking, this degrades the spatial resolution of the $34 \mathrm{GHz}$ image, which is comparable to that of the $17 \mathrm{GHz}$ image $\left(\sim 14^{\prime \prime}\right)$. Finally, we can derive a spectral index $\alpha$ by using the flux ratio, i.e., $\log \left(F_{34 \mathrm{GHz}} / F_{17 \mathrm{GHz}}\right)(\log (34 \mathrm{GHz} / 17 \mathrm{GHz}))^{-1}$.

Figure 3rc shows the map of the index $\alpha$ (we call it " $\alpha$-map") obtained with NoRH, overlaid with the NoRH $34 \mathrm{GHz}$ contour image with the white line. The core emission area is selected to be larger than $80 \%$ of the maximum intensity of $34 \mathrm{GHz}$ image, which corresponds to the inside region of the innermost (solid) contour line. We measure the spectral index $\alpha$ of the emission source, and found that it is about -3.0 , which is consistent with that derived from the NoRP spectrum. The center of the core emission region is $(-879$, $-241)$ arcsec heliocentric. The area is about $5.1 \times 10^{17} \mathrm{~cm}^{2}$ on the solar surface, and this corresponds to the solid angle $\omega$ of about $2.3 \times 10^{-9}$ str. The total fluxes of the region at $17 \mathrm{GHz}$ and at $34 \mathrm{GHz}$ are $F_{17} \sim 4.5 \times 10^{-19}$ and $F_{34} \sim 5.1 \times 10^{-20} \mathrm{erg} \mathrm{s}^{-1} \mathrm{~cm}^{-2} \mathrm{~Hz}^{-1}(4.5$ and $0.51 \mathrm{SFU})$, respectively. The maximum brightness temperature observed at the NoRH 
$17 \mathrm{GHz}$ is $3.1 \times 10^{6} \mathrm{~K}$ and that at the $34 \mathrm{GHz} 1.6 \times 10^{5} \mathrm{~K}$.

Here, we mention on the error to estimate the spectral index $\alpha$. The error is mainly caused by the relative displacement between the images at $17 \mathrm{GHz}$ and those at the $34 \mathrm{GHz}$ due to the NoRH image syntheses. The NoRH image syntheses hold an uncertainty of the positioning of about $5^{\prime \prime}$, and in this case, the error on $\alpha$ is about \pm 0.15 .

If we assume that the accelerated electrons follow a power-law distribution $d n_{\mu}(E) / d E=$ $K E^{-\delta_{\mu}}$ electrons $\mathrm{cm}^{-3} \mathrm{keV}^{-1}$ ( $K$ is a constant), the optically-thin gyrosynchrotron emission also follow a power-law distribution with the spectral index $\alpha$. There have been several studies to derive the relation between $\delta_{\mu}$ and $\alpha$, and we adopt the approximation derived by Dulk (1985) here. For the calculations, the number density of total electrons with higher energy than the lower-energy cutoff $E_{c}\left(E>E_{c}\right)$, that is, $N_{E_{c}}\left(=\int_{E_{c}}^{\infty} d n_{\mu}\left(E^{\prime}\right) / d E^{\prime} d E^{\prime}\right)$, is often used instead of $n_{\mu}(E)$, and Dulk (1985) assumed that $E_{c}=10 \mathrm{keV}$. The constant $K$ is also related to $N_{E_{c}}$ with the relation $K=(\delta-1) E_{c}^{\delta-1} N_{E_{c}}$. Dulk (1985) showed $F_{\nu} \propto \nu^{1.22-0.90 \delta_{\mu}}$, and therefore, we derive $\delta_{\mu} \sim(1.22-\alpha) / 0.90 \sim 4.7$ in the present case. From these, we can also estimate $N_{10 \mathrm{keV}}$ as follows:

$$
N_{10 \mathrm{keV}} \sim 3.4 \times 10^{42}(\sin \theta)^{-2.6} \frac{F_{\nu} \nu^{3.0}}{\omega l B^{4.0}} \sim 3.1 \times 10^{18}(\sin \theta)^{-2.6} B^{-4.0},
$$

where $\theta$ is the angle between the magnetic field $B$ and the line of sight, and $l$ is the line-ofsight depth of the emission source and is assumed here to be comparable to the width of the bundle of the EUV flare loops seen in the TRACE images, and about $20^{\prime \prime}\left(\sim 1.5 \times 10^{9} \mathrm{~cm}\right)$. Then, the constant $K$ is rewritten as $6.1 \times 10^{22}(\sin \theta)^{-2.6} B^{-4.0}$.

The gyrosynchrotron emission strongly depends on the magnetic field strength in the corona, which is very difficult to correctly measure. We use the microwave emission of the impulsive phase of the flare observed with NoRH to measure it. Fortunately, we observed the optically-thin thermal emission (White et al. 2003; Asai et al. 2006) in the before-the-flare phase (which corresponds to the Phase I in Paper I), which shows the circular polarization depending on the magnetic field strength (Dulk 1985). The degree of the circular polarization measured for the NoRH $17 \mathrm{GHz}$ emission source is about $1.6 \%$, which means $B_{0} \cos \theta \sim 48$ gauss. The estimation of $B_{0}$ depends on $\theta$, and becomes $\sim 190$ and 68 gauss for the cases of $\theta=75$ and $45^{\circ}$, respectively. For the further estimations in this paper, we used the $B_{0} \cos \theta$ measured in the before-the-flare phase as that in the preflare phase, although the magnetic field during the phase could be much different. If we assume that $B_{0}=150$ gauss and $\theta=71^{\circ}$. 0 , we found that

$$
\frac{d n_{\mu}(E)}{d E}=1.2 \times 10^{14} E^{-4.7}
$$

electrons $\mathrm{cm}^{-3} \mathrm{keV}^{-1}$. In this paper we assume that $E_{c}$ is $20 \mathrm{keV}$, and then, we got $N_{20 \mathrm{keV}} \sim$ 
$4.8 \times 10^{8}$ electrons $\mathrm{cm}^{-3}$. From the spectra of the HXR total flux, Holman et al. (2003) also derived $E_{c} \sim 20 \mathrm{keV}$ in this phase of the flare. We reported other cases with different magnetic field strengths $B=100,150$, and 200 gauss, and summarized the results in Table 1. We can easily confirm that the $N_{\mathrm{E}_{\mathrm{c}}}$ strongly depends on the magnetic field strength.

To generate the gyrosynchrotron emission observed in microwaves, such as at $17 \mathrm{GHz}$, with these magnetic field strengths (100 - 200 gauss), nonthermal electrons have to be accelerated to more than $1 \mathrm{MeV}$ (Kosugi et al. 1988; Bastian 1999). Therefore, we conclude that electrons are effectively accelerated to such high energy, even in the preflare phase of the flare. White et al. (2003) reported that the energy spectral index derived from NoRP, $\delta_{\mu}$ is about $2.7-1.8$ during the impulsive phase of the flare. However, these values could be underestimated due to the too high turn-over frequency which is reaching up to $30 \mathrm{GHz}$. On the other hand, the spectral index derived from the $40-400 \mathrm{keV} \mathrm{HXR,} \delta_{H}$ is about 4.5 during the peak time, which is almost the same as $\delta_{\mu}$ we obtained $(\sim 4.7)$. This implies that we observed the nonthermal electrons, which become the main component in the impulsive phase, even in the preflare phase.

\subsection{HXR Emission Source}

Second, we investigate the spectral index of the HXR emission source during the preflare by using the RHESSI data. In HXR range, we often observe nonthermal emission that follow a power-law distribution with the energy spectral index $\gamma$. The intensity $I(\epsilon)$ photons $\mathrm{s}^{-1} \mathrm{~cm}^{-1} \mathrm{keV}^{-1}$ is written as $I(\epsilon)=a \epsilon^{-\gamma}$, where $\epsilon$ is energy of the photon and $a$ is constant.

Here, we explain the way of the imaging spectroscopy of the HXR data. This method was originally suggested by Mitani (2005). We synthesized the HXR images in $2 \mathrm{keV}$-bin from 10 to $30 \mathrm{keV}$, and in $5 \mathrm{keV}$-bin from 30 to $50 \mathrm{keV}$. We integrated over 96 seconds, from 00:22:30 to 00:24:06 UT to synthesize the images in each energy bin. Figure 4 shows the image in each energy band. We can see that the loop top emission sources are dominant in each energy band. We can also see faint components elongated in the north-south direction in some images that probably outline the flare ribbons. Then, we draw a distribution histogram on the photon counts of an image in each energy band. We present an example in Figure 5 , which is the distribution histogram of the image in $20-22 \mathrm{keV}$ band. We fit the histogram with a Gaussian function, and determined the noise level of the image as $3 \sigma$ of the Gaussian function as shown in Figure 5. We selected the core emission region of the HXR images as shown with the box in Figure 4. All of the pixels within the core emission area are larger than the $3 \sigma$ level of the images from $10-12 \mathrm{keV}$ band to $40-45 \mathrm{keV}$ band. The center 
of the core region is $(-886,-239)$ arcsec heliocentric, where is slightly higher in vertical direction than the microwave core region. The image of $45-50 \mathrm{keV}$ is noisy and there are few pixels that exceeds the noise levels, so that we cannot distinguish the coronal emission source in the image. Therefore, we did not use the image for further spectral analyses.

The intensity of the core region clearly shows the power-law distribution as shown in Figure 6. We fitted the spectrum from 20 to $40 \mathrm{keV}$ range with a single power-law. As a result, we got $I(\epsilon)=3.2 \times 10^{6} \epsilon^{-\gamma}$, and $\gamma$ is $5.2 \pm 0.1$. We also showed the fitting result in Figure 6. The error bars on the plot show the $3 \sigma$ levels including the photon noise of the signal. This value $(\sim 5.2)$ roughly corresponds to the result that was derived from the spectroscopy of the total flux (no imaging) for the preflare phase of this flare (Holman et al. 2003), while their other results, i.e., the break of the spectra around $30 \mathrm{keV}$ and the softening at the higher energy range, are unclear in our observations. It may be because our result is based on the imaging spectroscopy from 10 to $40 \mathrm{keV}$, and the softening in the higher energy range does not appear clearly in the energy range. Alternatively, the single power-law fit that we derived may be just a weighted average of the spectral indices within this energy range, and the break and the steeper spectra at higher energy range may be missed. In addition, although the thermal component is also unclear in our result, it is consistent with the results by Lin et al. (2003b) and Holman et al. (2003) who reported that they could fit the spectra by the double power-law without any thermal components before 00:26 UT.

We could adopt the thin-target bremsstrahlung model to convert $\gamma$ into an electron power-law index $\delta_{H}$, since the HXR source is located on the loop-top, where is almost the same location as the nonthermal emission source seen in microwaves. Assuming the thintarget model for the HXR emission suggested by Hudson et al. (1978), the spectrum of the accelerated electrons $d n_{H}(E) V / d E$ electrons $\mathrm{keV}^{-1}$ ( $V$ is the volume of the emission source) is written as follows:

$$
\frac{d n_{H}(E) V}{d E}=1.05 \times 10^{42} \frac{a}{n_{i}} \frac{\gamma-1}{B\left(\gamma-1, \frac{1}{2}\right)} E^{-\gamma+0.5},
$$

where $E$ is energy of the electron, $n_{i}$ is the number density of ambient target plasma, and $B(p, q)$ is the Beta-function. Moreover, this model suggests that $d n_{H}(E) / d E=K^{\prime} E^{-\delta_{H}}\left(K^{\prime}\right.$ is a constant), and therefore, we found $\delta_{H}=\gamma-0.5 \sim 4.7$. Then, the electron spectrum is rewritten as $d n_{H}(E) / d E=2.7 \times 10^{49} n_{i}^{-1} V^{-1} E^{-4.7}$. The electron spectral index derived from the RHESSI HXR data $\delta_{H}$ is almost the same as that from the NoRH microwave data $\delta_{\mu}$. This implies that the distribution of the accelerated electrons follows a single power-law.

If the thin-target model is a reasonable assumption to the HXR emission source, we can further estimate the number density $d n_{H} / d E$ electrons $\mathrm{cm}^{-3} \mathrm{keV}^{-1}$ of the HXR-emitting electron in the core region. Assuming again that the width in the line-of-sight direction is 
comparable to the EUV flare loops seen in the TRACE images $\left(\sim 1.5 \times 10^{9} \mathrm{~cm}\right)$, the volume $V$ is estimated as $9.6 \times 10^{26} \mathrm{~cm}^{3}$. As we mentioned in $\S 2$, the density of the ambient plasma $n_{i}$ is roughly estimated from the GOES emission, and is about $2.4 \times 10^{10} \mathrm{~cm}^{-3}$. Therefore, we found that

$$
\frac{d n_{H}(E)}{d E}=1.2 \times 10^{12} E^{-4.7}
$$

electrons $\mathrm{cm}^{-3} \mathrm{keV}^{-1}$. Then, we also estimate the number density $N_{E_{c}}$ of nonthermal electrons with $E>E_{c}$. Since $N_{E_{c}}=\int_{E_{c}}^{\infty} d n_{H}\left(E^{\prime}\right) / d E^{\prime} d E^{\prime}$, the result strongly depends on the lower-energy cutoff $E_{c}$. Here, we assume the case of $E_{c}=20 \mathrm{keV}$, and we found that $N_{20 \mathrm{keV}}=5.1 \times 10^{6}$ electrons $\mathrm{cm}^{-3}$. These results on the accelerated electrons are about two orders of magnitude less than those from the microwave emission (c.f. eq. 2). Therefore, it may be better to consider that the emissions showed the same spectral indices by an accidental coincidence.

Then, we consider the thick-target model to explain the HXR emission source. The accelerated nonthermal electrons seem to be effectively trapped in the corona in this phase, since we cannot clearly see footpoint sources but loop-top sources (see Fig. 3). Therefore, we suggest that the trapped nonthermal electrons are exhausted in the corona with the thicktarget model, and apply the trap and precipitation model or the thick-thin target model (Melrose \& Brown 1976; Aschwanden 1998; Wheatland \& Melrose 1995; Metcalf \& Alexander 1999). The thick-thin target model suggests that a break, which increases with time from about $15 \mathrm{keV}$ to $40 \mathrm{keV}$ or more, in a HXR spectrum, and thick-(thin-) target model should be adopted below (above) the break energy. Holman et al. (2003) reported that there is a break of the total photon spectra at around $30 \mathrm{keV}$, which probably shows the transitions from the thick-target to the thin-target, while it is unclear in our imaging spectroscopic study. Following the eq. 1 of Metcalf \& Alexander (1999), the break energy is estimated $E_{t}=20 \times\left(n_{i} l_{e} / 10^{20}\right)^{0.5}\left(0.7 / \cos \alpha_{0}\right)^{0.5} \mathrm{keV}$, where $l_{e}$ is the length that an accelerated electron travels, and $\alpha_{0}$ is the average pitch angle of the trapped electrons. We roughly estimate that $l_{e}$ is twice as long as the height of the flare loops seen in the TRACE images (i.e., we assume that the the X-point is located twice as high as the loop height, and that the accelerated electrons are thermalized before they bombard at the footpoints), and is about $80^{\prime \prime}\left(\sim 5.8 \times 10^{9} \mathrm{~cm}\right)$. Therefore, we get $E_{t} \sim 24 \times\left(0.7 / \cos \alpha_{0}\right)^{0.5} \mathrm{keV}$, and it is, for example, about $34 \mathrm{keV}$ for $\alpha_{0} \sim 70^{\circ}$.

In the case of the thick-target model, the power-law distribution of the injected electron flux $F(E)$ electrons $\mathrm{s}^{-1} \mathrm{keV}^{-1}$ is written as (Hudson et al. 1978):

$$
F(E)=\frac{d^{2} n_{H}(E) V}{d E d t}=3.28 \times 10^{33} a \gamma^{2}(\gamma-1)^{2} B\left(\gamma-\frac{1}{2}, \frac{3}{2}\right) E^{-\gamma-1.0}
$$


In the present case we get

$$
F(E)=\frac{d^{2} n_{H}(E) V}{d E d t}=7.0 \times 10^{41} E^{-6.2},
$$

and the spectral index $\delta_{H}$ is about 6.2. Moreover, we get $d n_{H}(E) / d E \sim F(E) \tau V^{-1}=$ $5.8 \times 10^{14} E^{-6.7}$ electrons $\mathrm{cm}^{-3} \mathrm{keV}^{-1}$, assuming that $\tau$ is the electron traveling time and $\tau \sim l v_{e}=l \sqrt{m_{e} /(2 E)}$, where $v_{e}$ and $m_{e}$ are electron velocity and mass, and the traveling length $l$ (which is assumed to be $1.5 \times 10^{9} \mathrm{~cm}$ ). In this case the total number of the accelerated electrons $\left(N_{20 \mathrm{keV}}=4.0 \times 10^{6}\right.$ electrons $\left.\mathrm{cm}^{-3}\right)$ is somewhat closer to that derived from the microwave than those obtained in the thin-target case, while the spectral index $\delta_{H}(\sim 6.7)$ is much different from those for the microwave-emitting electrons.

In the impulsive phase of the flare, the RHESSI HXR spectra are well fitted with thermal plus double power-law distributions (Holman et al. 2003; White et al. 2003). Especially, the coronal source no longer shows the nonthermal features, and is responsible for the thermal component in the spectrum (Emslie et al. 2003). The power-law components, which mainly come from the footpoint sources, show a quite harder spectrum with $\gamma \sim 3$ than that for the preflare phase, and the corresponding electron energy spectral index is about $\delta \sim 4.5$, assuming the thick-target model for the HXR emission. As we mentioned above, this is very close to the spectral index derived from the microwave emission in the preflare phase $(\sim 4.7)$.

\section{Summary and Discussions}

We performed imaging spectroscopic analyses on the emission sources observed both in NoRH microwaves and in RHESSI HXRs. Both the emission sources are located above the post flare loops in the corona, although the HXR emission source is located slightly higher, and they clearly show nonthermal features. Based on the gyrosynchrotron theory (Kosugi et al. 1988; Bastian 1999), nonthermal electrons have to be accelerated to higher than $1 \mathrm{MeV}$ even in this phase.

If we assume the thin-target model for the HXR emission source, the electron spectral index $\delta_{H}$ of about 4.7 showed the same value as that from microwaves $\delta_{\mu}(\sim 4.7)$ within the observational uncertainties. This result implies that the distribution of the accelerated electrons follows a single power-law. The number density of the nonthermal electrons that emit the microwaves is, however, much larger than that of the HXR-emitting electrons.

If the real magnetic field strength is higher than our estimation, the gap between these indices reduces. For example, if we assume very strong magnetic field of about 300 gauss $\left(\theta=81^{\circ}\right)$, then, $N_{20 \mathrm{keV}}=2.7 \times 10^{7}$, which is somewhat close to the result derived from the 
thin-target model. However, as White et al. (2003) reported, such high magnetic field is not expected in the impulsive phase of the flare from the gyrosynchrotron theory (Dulk \& Marsh 1982), and they also commented that magnetic field strength is probably no more than 200 gauss. Although we cannot directly adopt the same discussion to the preflare phase, magnetic field strength of about 300 gauss is somewhat unusual. Furthermore, the TRACE $195 \AA$ images do not show that the flare loops stand in a direction exactly perpendicular to the line-of-sight. Therefore, the larger $\theta$ (such as $80^{\circ}$ or more), and therefore, the strong magnetic field cannot be expected.

We may overestimate the ambient plasma density. If we assume the number density of about $n_{i}=1 \times 10^{9} \mathrm{~cm}^{-3}$, we found that $N_{20 \mathrm{keV}}=1.2 \times 10^{8}$ electrons $\mathrm{cm}^{-3}$ for the thin-target models. These are comparable to the results from $n_{\mu}$ with the case of $B=200$ gauss (see Table 1). On the other hand, we can see faint flare loops and two-ribbon structure in TRACE images, which implies that hot plasma with the temperature of about $20 \mathrm{MK}$ fills the whole arcade region, even in the preflare phase. Then, we can estimate the maximum size of the hot plasma region to be about 40" (height) $\times 40^{\prime \prime}$ (length) $\times 20^{\prime \prime}$ (width) $\left(\sim 1.2 \times 10^{28} \mathrm{~cm}^{3}\right)$, and obtain the minimum density of about $1.4 \times 10^{10} \mathrm{~cm}^{-3}$, which is not so small. Therefore, the number density of about $10^{9} \mathrm{~cm}^{-3}$ seems too small based on the observations, although we cannot discard the possibility of such small ambient plasma density at above-the-loop top region where the HXR emission source appeared.

The actual HXR spectra may break at about $30 \mathrm{keV}$ and steepen at the higher energy range, which Holman et al. (2003) reported in their spatially integrated analysis, although it is unclear in our result. (A HXR detector with a greater collecting area than that of RHESSI's so that useful images can be obtained to higher energies and narrower energy bands can be used would be a highly desirable feature for a future instrument to make clear the issue.) If such broken spectra are entirely applicable to the coronal HXR emission source, it could be explained by the thick-thin model with the electron spectral index $\delta$ of about 6.5 , and the thick-target should be responsible for the observed HXR emission. Moreover, in that case, we may explain the gap of the spectral index derived from the thick-thin HXR emission $\left(\delta_{H} \sim 6.5\right)$ and that from the microwave $\left(\delta_{\mu} \sim 4.7\right)$ at higher energy ranges, by considering that we may underestimate the thermal component in microwaves. Especially, the microwave emission at $34 \mathrm{GHz}$ may suffer more from the thermal contribution from cooler plasmas than detected by GOES. This gives a steeper microwave spectra than that estimated in this paper. To settle the uncertainty of the microwave spectral index, a microwave interferometer with much high spectral resolution up to $40 \mathrm{GHz}$ or more is required. However, even though, it seems that such a large electron spectral index $\delta_{\mu}$ of about 6.5 is unexpected in the present case. 
We finally have to note the difference of the positions of the microwave and HXR emission sources. The HXR emission source is located slightly higher than the microwave emission source, as is often reported, and therefore, it is possible that the HXR-emitting electrons are belong to the different group from that for the microwave-emitting electrons. The displacement between the microwave and HXR coronal emission sources has been known for many flares, and the magnetic field strength at the source position has been discussed to explain this. Loop-top HXR sources are probably located above the SXR flare loops, and therefore, the magnetic field there is weaker than the top of closed flare loops. Statistical studies on the displacement will be discussed in our future papers.

We first acknowledge an anonymous referee for her/his useful comments and suggestions. We wish to thank Drs. M. R. Kundu, E. J. Schmahl for fruitful discussions and their helpful comments. This work was carried out by the joint research program of the SolarTerrestrial Environment Laboratory, Nagoya University. We made extensive use of TRACE and RHESSI Data Center.

\section{REFERENCES}

Asai, A., Nakajima, H., Shimojo, M., White, S. M., Hudson, H. S., Lin, R. P. 2006, PASJ, 58, L1

Aschwanden, M. J. 1998, ApJ, 502, 455

Aschwanden, M. J. 2002, Space Sci. Rev., 101, 1

Bastian, T. S. 1998, in NRO Report 479, Solar Physics with Radio Observations, ed. T. Bastian, N. Gopalswamy, \& K. Shibasaki (Nagano: Nobeyama Radio Observatory, NAOJ), 211

Dulk, G. A. \& Marsh, K. A. 1982, ApJ, 259, 350

Dulk, G. A. 1985, ARA\&A, 23, 169

Emslie, G. A., Kontar, E. P., Krucker, S., Lin, R. P. 2003, ApJ, 595, L107

Handy, B. N., et al. 1999, Sol. Phys., 187, 229

Holman, G. D., Sui, L., Schwartz, R. A. \& Emslie, A. G. 2003, ApJ, 595, L97

Hudson, H. S., Canfield, R. C., \& Kane, S. R. 1978, Sol. Phys., 60, 137 
Kosugi, T., Dennis, B. R., \& Kai, K. 1988, ApJ, 324, 1118

Kundu, M. R. 1961, J. Geophys. Res., 66, 4308

Lin, R. P., et al. 2002, Sol. Phys., 210, 3

Lin, R. P., et al. 2003a, ApJ, 595, L69

Lin, R. P., Krucker, S., Holman, G. D., Sui, L., Hurford, G. J., \& Schwartz, R. A. 2003b, in Proc. 28th Int. Cosmic Ray Conf. (Tsukuba), 3207

Melrose, D. B., Brown, J. C. 1976, MNRAS, 176, 15

Metcalf, T. R. \& Alexander D. 1999, ApJ, 522, 1108

Mitani, N. 2005, Master thesis, Univ. Tokyo (in Japanese)

Nakajima, H., Sekiguchi, H., Sawa, M., Kai, K., Kawashima, S. 1985, PASJ, 37, 163

Nakajima, H., et al. 1994, in Proc. of the IEEE, 82, 705

Schrijver, C. J., et al. 1999, Sol. Phys., 187, 261

Shibasaki, K., Ishiguro, M., \& Enome, S. 1979, Proc. Res. Inst. Atmos., 26, 117

Silva, A. V. R., Gary, D. E., White, S. M., Lin, R. P., \& de Pater, I. 1997, Sol. Phys., 175, 157

Somov, B. V, \& Kosugi, T. 1997, ApJ, 485, 859

Spitzer, L. 1962, Physics of Fully Ionized Gases (2nd ed.; New York: Wiley)

Torii, C., Tsukiji, Y., Kobayashi, S., Yoshimi, N., Tanaka, H., \& Enome, S. 1979, Proc. Res. Inst. Atmos., 26, 129

Wheatland, M. S., \& Melrose, D. B. 1995, Sol. Phys., 185, 283

White, S. M., Krucker, S., Shibasaki, K., Yokoyama, T., Shimojo, M., Kundu, M. R. 2003, ApJ, 595, L111 
Table 1: Parameter survey for microwave emission source

\begin{tabular}{llll}
\hline \hline $\begin{array}{l}B_{0} \\
\text { gauss }\end{array}$ & $K$ constant $^{a}$ & $\begin{array}{l}\theta \\
\text { deg }\end{array}$ & $\begin{array}{l}N_{20 k e V} \\
\text { electrons cm }\end{array}$ \\
\hline 100 & $7.8 \times 10^{14}$ & 60.7 & $3.1 \times 10^{9}$ \\
150 & $1.2 \times 10^{14}$ & 71.0 & $4.8 \times 10^{8}$ \\
200 & $3.6 \times 10^{13}$ & 75.8 & $1.4 \times 10^{8}$ \\
\hline
\end{tabular}

${ }^{a} d n_{\mu}(E) / d E=K E^{-\delta_{\mu}}$ electrons $\mathrm{cm}^{-3}$ 

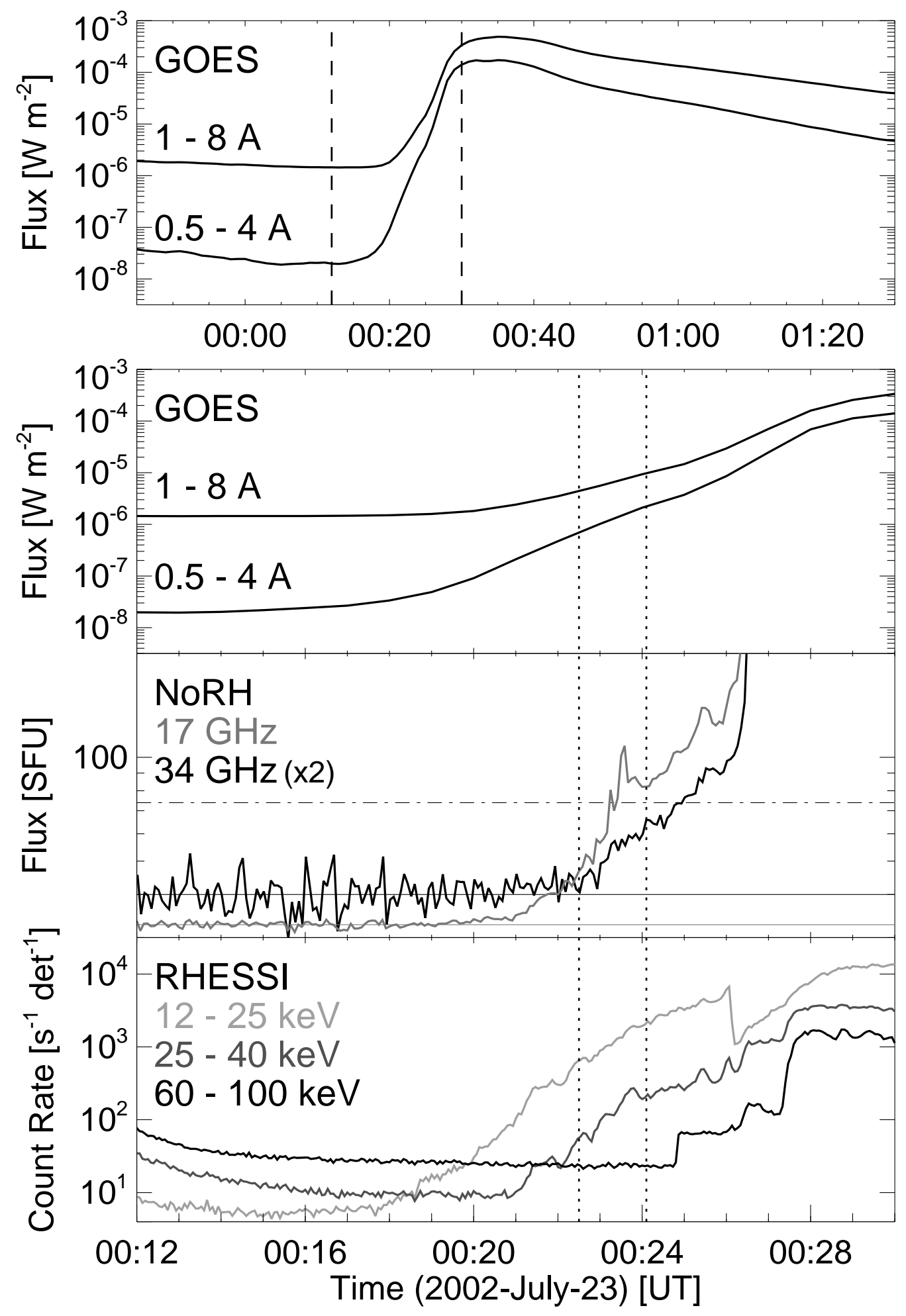

Fig. 1. - Lightcurves of the 2002 July 23 flare. From top to bottom: soft X-ray flux in the GOES 1.0 - $8.0 \AA$ channel; the same plot, but scaled up around the preflare phase that are indicated with two dashed vertical lines in the top; radio flux observed at $17 \mathrm{GHz}$ (gray) and $34 \mathrm{GHz}$ (black) by NoRH; HXR count rate measured by RHESSI in $12-25 \mathrm{keV}$ (light gray) , $25-40 \mathrm{keV}$ (dark gray), and $60-100 \mathrm{keV}$ (black). Two dotted vertical lines show the time range for the integration of the RHESSI image synthesis. The NoRH $34 \mathrm{GHz}$ flux in the third panel is multiplied by 2 , and re-scaled so that the average flux, which was $73.8 \mathrm{SFU}$ 


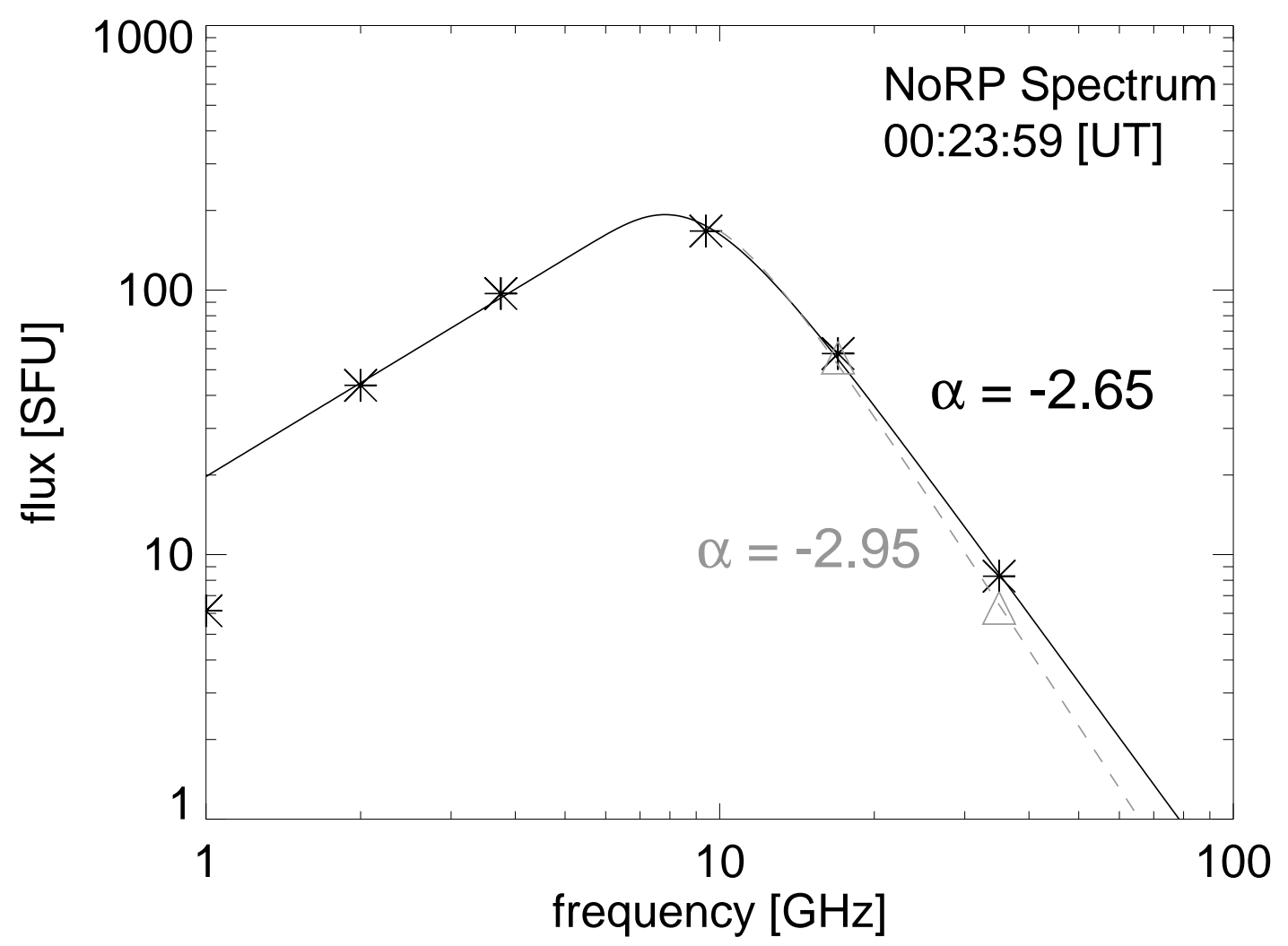

Fig. 2.- Spectrum taken at 00:23:59 UT by NoRP. The fitted parameters are as follows: the index $\alpha$ for the optically-thick part is 1.17, that for the optically-thin part is -2.65 , the turn-over frequency is $9.4 \mathrm{GHz}$, and the peak flux is 276 SFU. Subtracting the thermal microwave emission, which is about $2 \mathrm{SFU}$, we re-plotted the spectrum with the index $\alpha$ for the optically-thin part of -2.95 , as shown with the gray dashed line. 


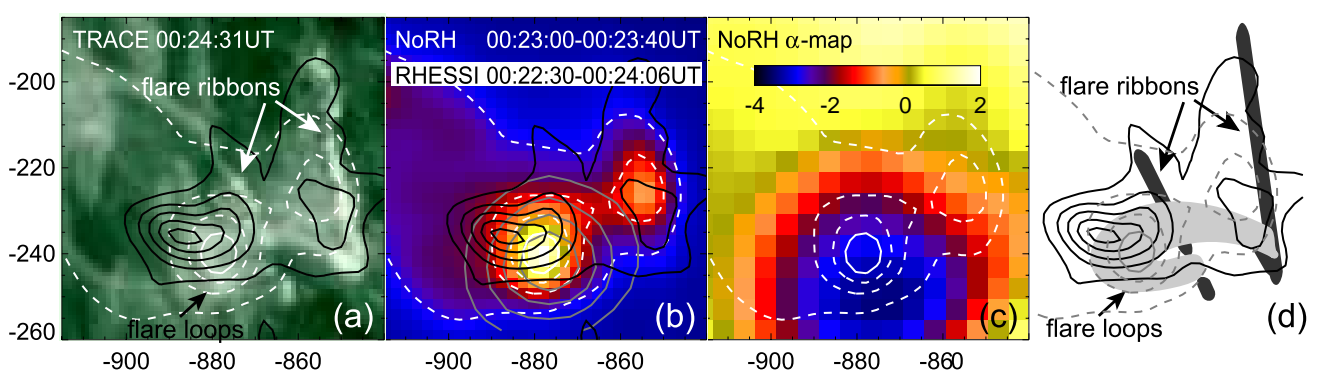

Fig. 3.- Images for the preflare phase of the flare. Solar north is up, and west is to the right. (a) shows the EUV image taken with TRACE $195 \AA$ at 00:24:31 UT. (b) (c) show the microwave image of NoRH $34 \mathrm{GHz}$ and the map of NoRH $\alpha$ index. (d) shows the positions of the flare ribbons (dark gray regions) and the flare loops (light gray). On each panel we overlaid the contour images of NoRH $34 \mathrm{GHz}$ with the white dashed line (with the gray dashed line for d). The NoRH $17 \mathrm{GHz}$ contour image is further overlaid on the panel (b) with the gray solid line. The levels of the NoRH contours are 20, 40, 60, and $80 \%$ of the peak intensity. We also showed the core region of the microwave loop-top emission source with the white solid line that is the $80 \%$ contour line. We also overlaid the contour images of the RHESSI 30 - $40 \mathrm{keV}$ intensity and black solid line on (a), (b), and (d). The RHESSI contours are $20,40,60,80$, and $95 \%$ of the peak intensity. 

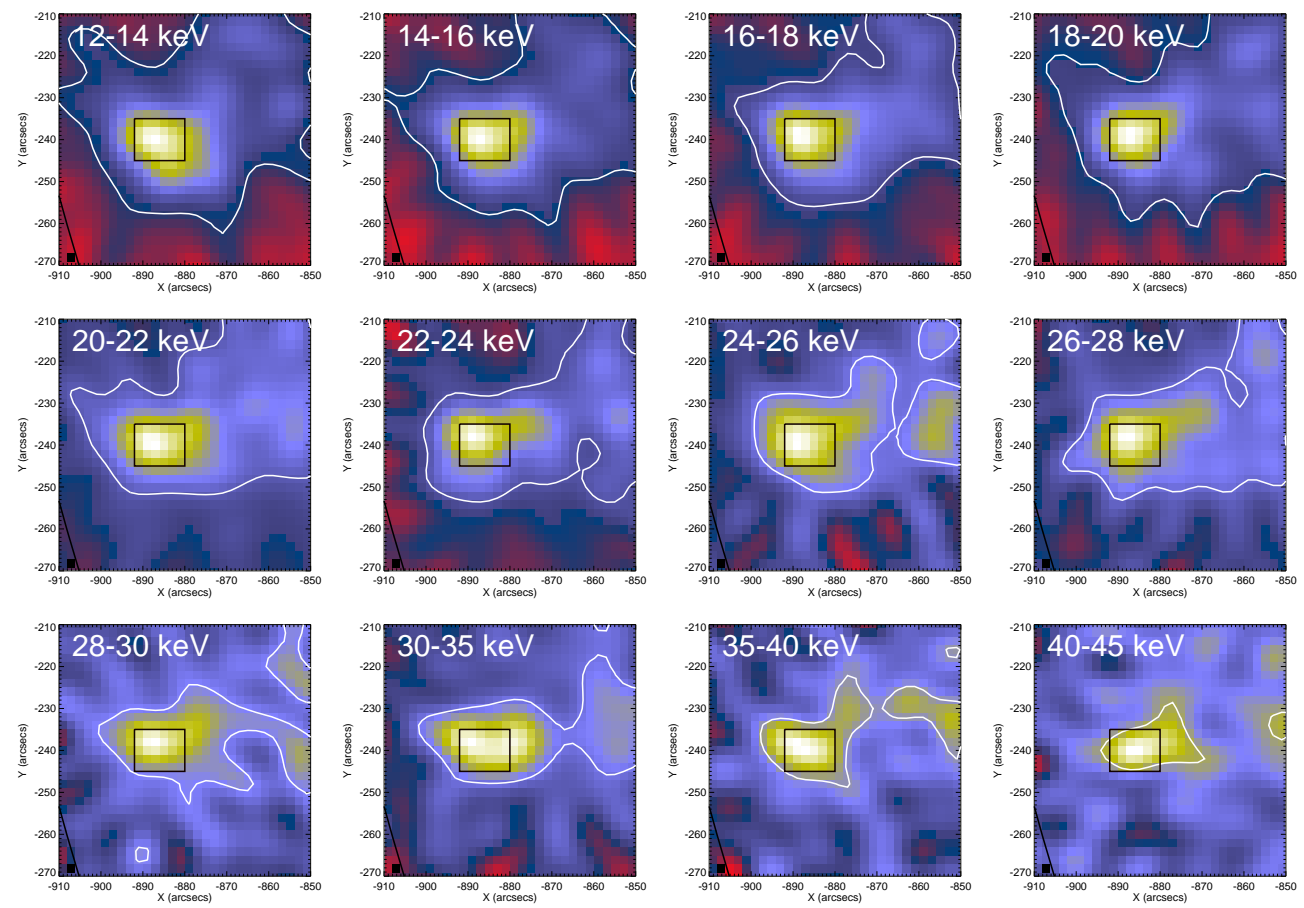

Fig. 4.- HXR images in each energy bin from 12 to $45 \mathrm{keV}$. The rectangles with the black lines show the core emission region. The white contour lines show $3 \sigma$ level of each HXR image. 


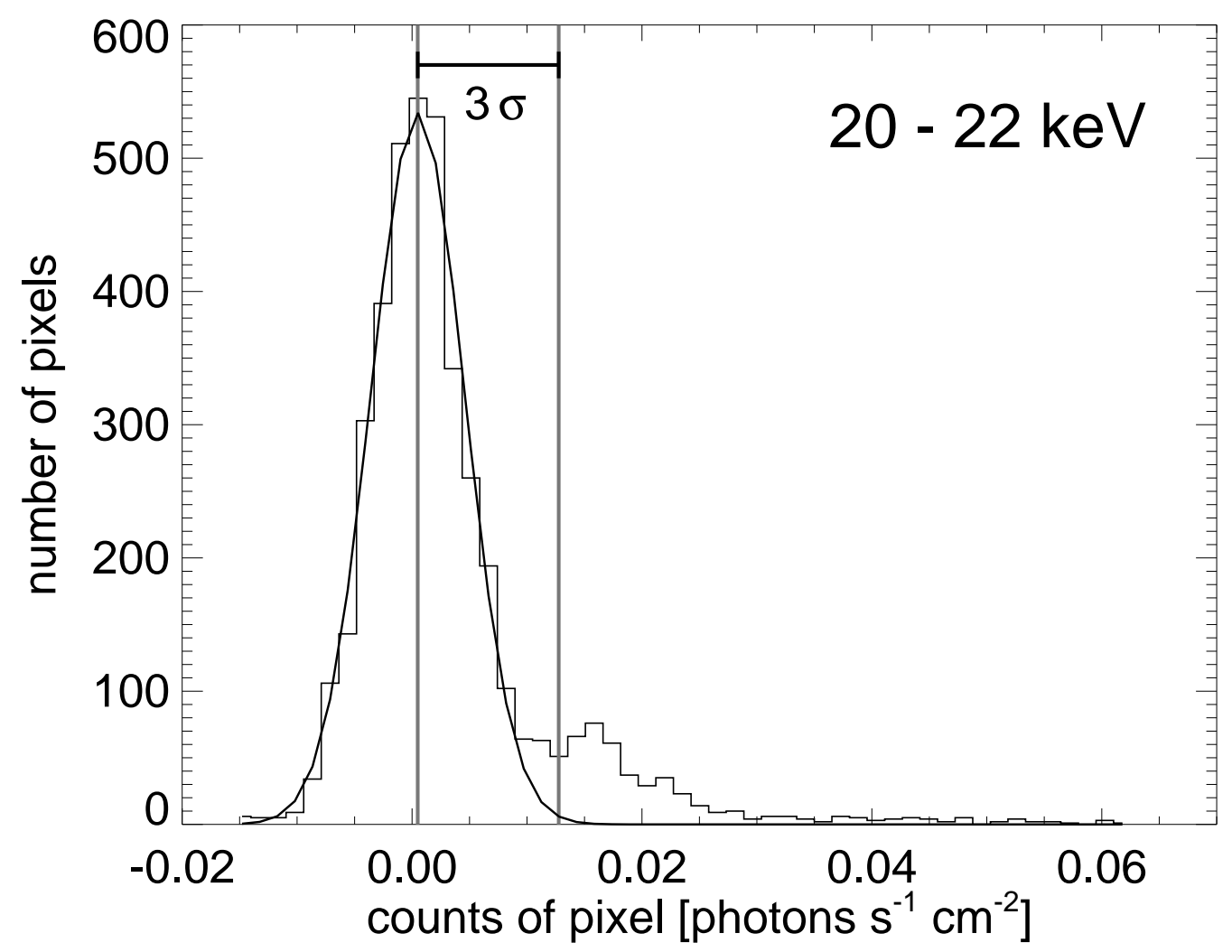

Fig. 5.- Distribution histogram of the RHESSI image in $20-22 \mathrm{keV}$ band. We overlaid Gaussian function with the solid line. The $3 \sigma$ level of the Gaussian function is also shown with the vertical line. 


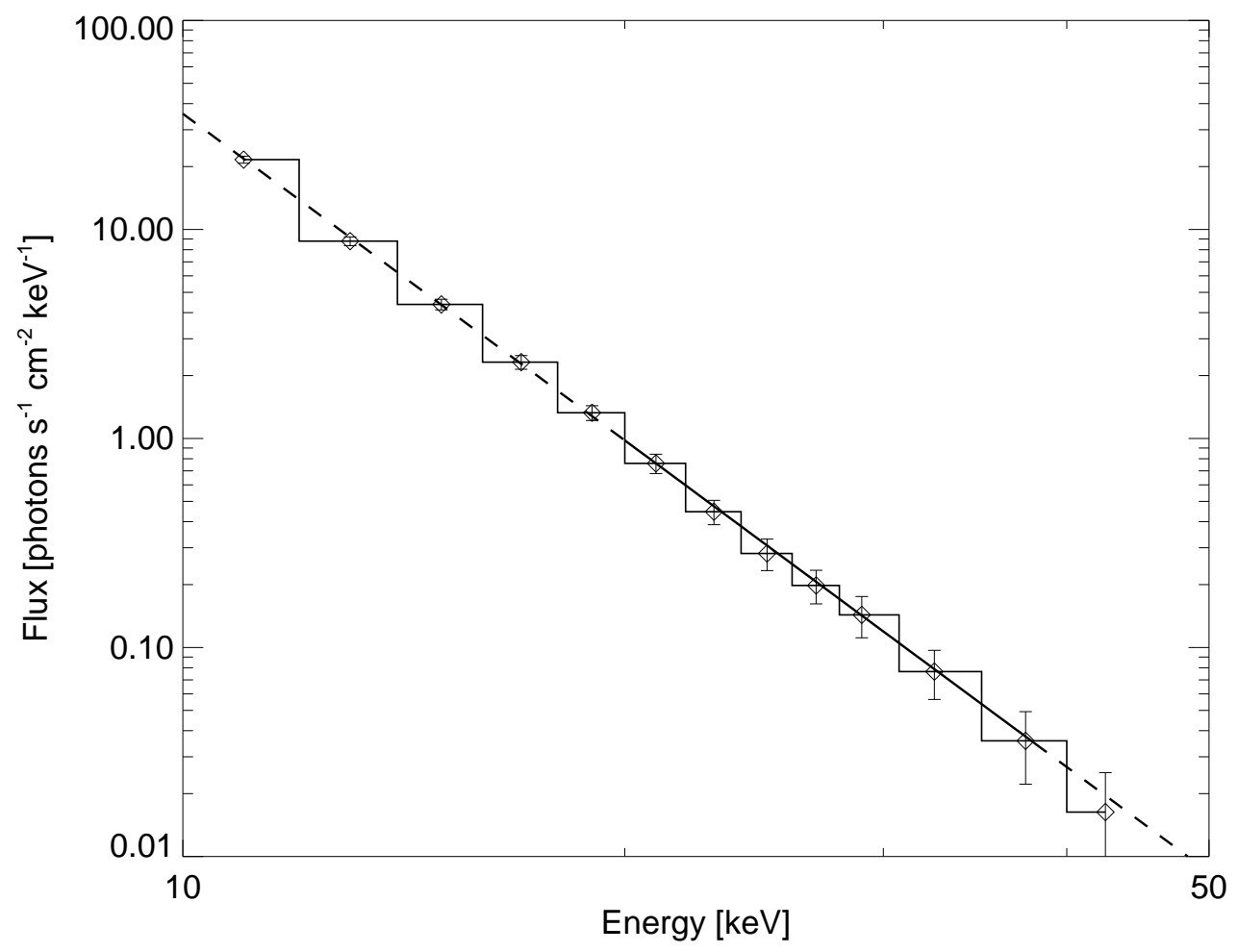

Fig. 6. - Photon spectrum of HXR emission region overlaid with the fitting plot. The time interval is from 00:22:30 to 00:24:06 UT (96 sec). The counts for the fit are integrated over the core emission region shown in Figure 4 . The error bars show the quadrature of the $3 \sigma$ level of each energy bin and the $3 \sigma$ level of the photon noise. 